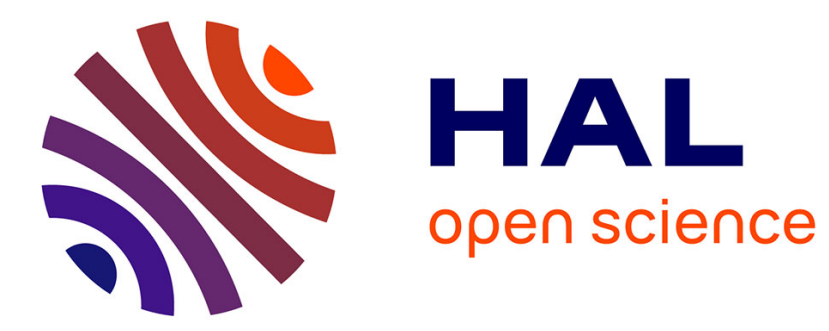

\title{
Change management of BPM-based software applications
}

\author{
Mourad Bouneffa, Adeel Ahmad
}

\section{To cite this version:}

Mourad Bouneffa, Adeel Ahmad. Change management of BPM-based software applications. International Conference on Enterprise Information Systems (ICEIS), 2013, Angers, France. hal-01894065

\section{HAL Id: hal-01894065 \\ https://hal.science/hal-01894065}

Submitted on 12 Oct 2018

HAL is a multi-disciplinary open access archive for the deposit and dissemination of scientific research documents, whether they are published or not. The documents may come from teaching and research institutions in France or abroad, or from public or private research centers.
L'archive ouverte pluridisciplinaire HAL, est destinée au dépôt et à la diffusion de documents scientifiques de niveau recherche, publiés ou non, émanant des établissements d'enseignement et de recherche français ou étrangers, des laboratoires publics ou privés. 


\title{
Change management of BPM-based software applications
}

\author{
Mourad Bouneffa, Adeel Ahmad \\ Université Lille Nord de France \\ Laboratoire d'Informatique Signal et Image de la Côte d'Opale \\ 50, rue Ferdinand Buisson BP 71962228 Calais Cedex France \\ \{bouneffa,ahmad\}@lisic.univ-littoral.fr
}

Keywords: $\quad$ BPM, Software Evolution, Change impact propagation, Graph rewriting Rules, Process change management

\begin{abstract}
Business process models are decision making tools intended to describe operations, constraints, and policies of an organisation. In this paper, we present an approach to control the evolution of Business Process Model (BPM) based software applications. We define a BPM change taxonomy and focus on the change affecting the BPMs and its impact propagation, through traceability relationships, to the software applications implementing them. From a formal point of view, our work consists of specifying a BPM meta-model using a typed attributed graph and a BPM change operations taxonomy based on graph rewriting rules. The specifications are then implemented using an integrated software change management platform appearing as a set of the Eclipse Workbench plug-ins.
\end{abstract}

\section{INTRODUCTION}

The communication means and interfaces between human users and the automated information systems are rapidly developing for the past decade. Thus, the idea to automate most of the information system processes, destined to managing an organization, is far from being a utopia. It is therefore possible for an external user of the organization to communicate with the information system of such an organization through various types of communication media and interfaces such as voice systems, applications running on smart-phones, etc. Moreover, these entry points to information systems can be provided by the various devices of operational systems to facilitate a coherent integration of the different organizational actors, management processes, and software applications supporting these processes.

This leads to the emergence of new software development tools and approaches based on the Business Process Model(BPM)(Weske, 2007; Weske, 2012) concept. In these approaches the BPMs are specified by means of some standard notations like BPMN (Silver, 2009; Allweyer, 2010) and XPDL (Van der Aalst, 2003; Haller et al., 2008). The BPMs are then transformed into executable programs that are generally deployed as multi-tiered distributed applications using platforms like J2EE, .NET, etc. The executable programs are often built as macro programs implementing the well known con- cept of programming in the large (Emig et al., 2005). These programs contain invocations of web services (Gottschalk et al., 2002) provided by the various software applications which have been deployed inside or sometimes outside the information system boundaries. The Business Process Execution Language (BPEL) (Juric, 2006) is one of the most known programming in the large language.

In this paper, we precisely consider the study of this new generation of applications. Our goal is to demonstrate the feasibility of the implementation of a process to control the change impact which may affect these applications. For this purpose, we elaborate a meta-model permitting to represent the most significant elements of a BPM. The major objective is to formally construct a structure to express the several BPM change operations.

The meta-model is formalized using typed and attributed graphs. Our modelling choice of formally specifying BPM allows to describe change operations using graph rewriting or transformation rules. Such rules can, indeed, permit a visual definition of the effective change operations. These are adapted to study the software artifact changes which are often expressed by the graph change operations. A taxonomy is then elaborated to define the construction rules and BPM transformations. The obtained specification is aimed at defining a change impact not only on BPM itself but also on concerned components of the BPM 
based software applications. Our goal includes the traceability between software applications, deployment platforms, software system actors and BPMs. The main objective being to control the change impact propagation through all, or a specified part of such a software system.

In Section 2 we present, the meta-model for the BPM formalization and the notions relevant to BPM and BPM-based software applications. In section 3 , we specify a taxonomy of BPM change operations and we give the formalization of these operations by the graph rewriting rules. The section 4 presents the change impact analysis and propagation process along with its formalization by the graph rewriting rules. The section 5 shows the prototype implementation of our specifications in regard to the integrated platform to control the software changes(M.O. et al., 2010). The section 6, summarizes the contribution by a conclusion with the different perspectives of this work.

\section{BPM FORMALIZATION AND META-MODELING}

Before explaining the formalization and metamodeling of BPM, we first explain the concept of BPM and especially the life cycle of software applications based on the BPM. As shown in Fig. 1, the development, deployment, and evolution of BPM based software applications obey a life cycle. The phases of BPM life cycle are described in the following sections :

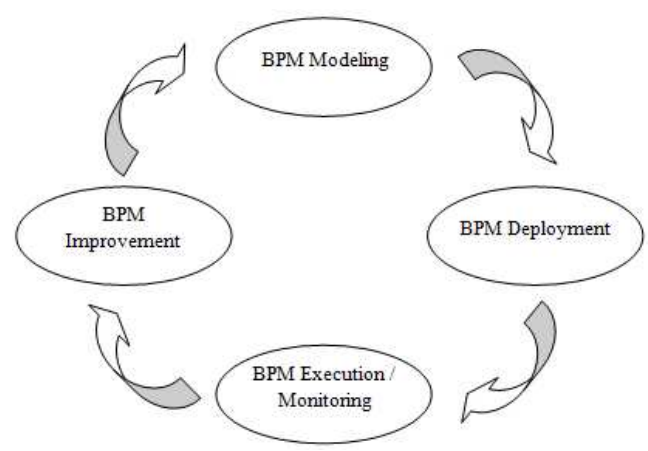

Figure 1: The life cycle of BPM based applications

\subsection{The BPM Modeling}

This phase involves BPM Modeling in terms of tasks or activities which are necessary to implement the process, the order of tasks accomplishment, the human actors involved in the performance of these tasks, etc. In recent years, several models or notations have been defined to model the BPM. At first, designing a BPM is an activity within the scope of the information system cartography. It was most often performed as a part of BPR (Business Process Re-engineering) projects (Lee et al., 2011). Thus, many models and methods have been implemented such as the OSSAD method(Dumas et al., 1990), etc. Our present work is particularly related to the BPM as a means of specification, development, and deployment of automated processes. We selected the widely considered and used notations in this area, particularly the BPMN (OMG, 2011). Fig. 2 shows an example of such a process. In this figure, the process is a partial description of the Sales Chain Management. We first distinguish two important actors : the Client and the Process Order. At the beginning, a start event represent the fact that Place Order is the first task. This first task performed by the Client consists of the generation of an order that is sent as a message to the Check Availability task, which is linked to a gateway involving the Check Payment task. If the products are available or the Cancel Order otherwise, etc. The process ends by end events linked with Confirm Order and Cancel Order tasks.

\subsection{The Development and the deployment of the BPM}

The development and the deployment of a BPM are two separate activities that can be performed manually, automatically, or usually semi-automatically. In principle, these activities can be considered as classical operations for code generation, where the BPM plays the role of a detailed design and the code is represented by an application, deployed most often on a web platform. There exists also software tools to automate the deployment of such applications almost transparently. Some of these tools are Bonita ${ }^{1}$, Intalio $^{2}, \mathrm{BizAgi}^{3}$ and Barium Live! ${ }^{4}$, etc. In these tools a web application is generated and is hosted by a Java servlet engine or in the form of ASP.NET pages, etc. Without going to the full automation and complete transparent development and deployment of BPM based applications, there are intermediate languages playing the role of orchestrators or macro programs involving software components already encapsulated by web services. BPEL is one of the main

\footnotetext{
${ }^{1}$ Bonita Open Solution url: http://www.bonitasoft.com/

${ }^{2}$ Intalio-BPMS : http: //www.intalio.com/

${ }^{3}$ Bizagi BPM Suite: http://www.bizagi.com/

${ }^{4}$ Barium Live! : http: / / www . bariumlive.com/
} 


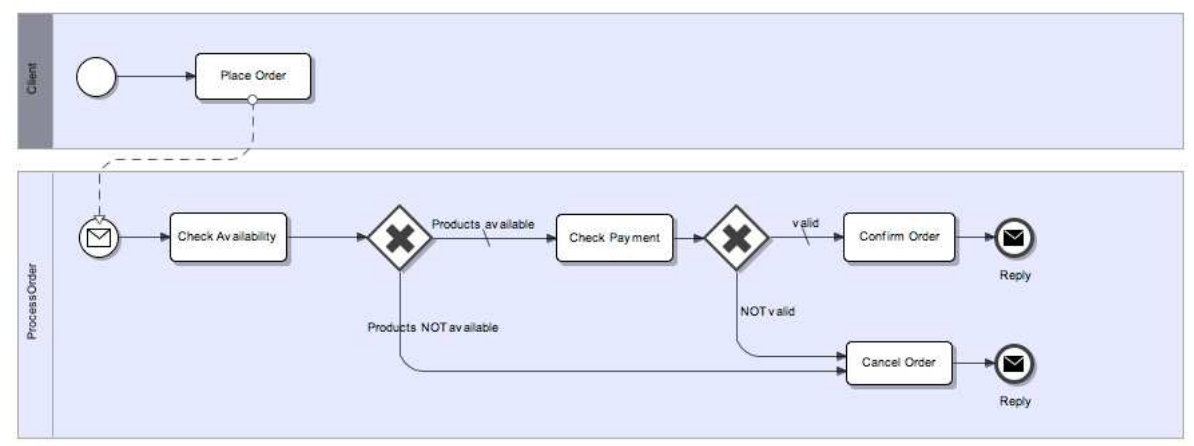

Figure 2: An example of Business Process Model Notation

languages of this type and appears like a sort of standard in the matter. In one perspective of Model Driven Engineering (MDE) (Schmidt, 2006), BPMN can be considered as a Platform Independent Model (PIM) and BPEL as a Platform Specific Model (PSM) consisting of implementing BPMN in an environment using web services as a means of communication and interoperability.

Fig. 3 shows an example of BPEL implementing the BPM shown in Fig. 2. In this figure the BPEL is a kind of web services orchestration. To do this, the BPEL contains calls or invocations to web

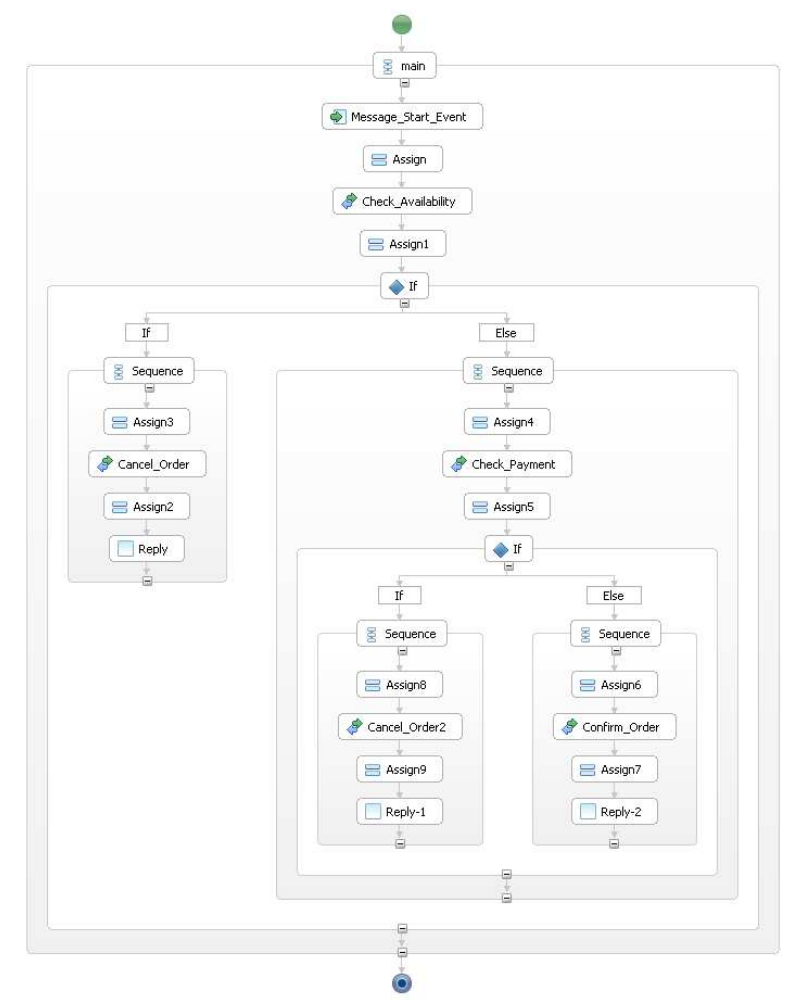

Figure 3: An example of Business Process Execution Language services like Check Availability, Check Payment and Cancel Order and flow management nodes like sequence for a sequential execution of web services invocations or If for conditional branches. It is useful to remark that the programming in the large concepts are quite similar to the programming in the small (DeRemer and Kron, 1975) ones. For our example, the tasks or activities of the BPMs are implemented by web service calls while gateways are implemented by If nodes.

\subsection{The execution and monitoring of the BPM}

The execution of the application is generally assured by a web platform which is usually on multi-tiered architecture etc. The BPM execution provides generally some interesting data such as response time, resource consumption, etc. These data are necessary for the purpose of analyzing the process quality. Indeed, business managers define performance indicators (Key Performance Indicator (Parmenter, 2007)) for each process, to measure the performance of activities implemented by processes. Some of these informations can be obtained by dynamic analysis by means of program profiling techniques (Ahmad et al., 2008b; Ahmad and Basson, 2009; $\quad$ Ahmad et al., 2009). Other information are exclusively provided by human experts and will be explicitly taken into account by some organizational process. They are generally the derived data from activities within the framework of customer satisfaction, etc. In (Dadam et al., 2007), the authors propose, during the execution of processes, to consider particularly the exceptions. In some other areas, such as medical processes, it is not possible to foresee all possible cases and to model them through the processes. Thus, the authors in (Dadam et al., 2007) propose and implement an exception handler, able to propose an alternative implementation or implementations of processes on 
the fly. A system of corporate knowledge about exceptions can then eventually propose improvements to the initial process to take into account the various exceptions.

\subsection{The BPM improvement}

The process improvement is a generic term that refers primarily to the evolution of BPM processes. In reality, the improvement is expected but what is actually done, is an evolution of a process embodied by the change affecting the BPM processes. The goal is to fix certain performance anomalies or simply to complete the automation of processes, a part of which is manual, etc. In the literature, the improvement is seen only on the process side and it ignores the software implementation problems. In our case, we consider both aspects, analyzing in particular the BPM change impacts on the software and vice versa.

\subsection{A meta-model of graph-based BPM}

We propose a meta-model to represent the concepts involved in the definition of BPMs. This meta-model has been formalized by a typed graph that we implement particularly in the context of the $\mathrm{AGG}^{5}$. The result of this modeling is shown schematically in the Fig. 4. This figure represents the main concepts emerging from the BPMN. The process concept represents the processes that contain what is called flow objects. These objects can be tasks or activities, sub-processes or macro-tasks, refined by the processes, events or gateways. A process has an actor which is called the owner which may be one user, or more, who has defined the process and is authorized to change this process. The process is implemented by an application which is deployed and which can host the execution of multiple instances or cases of this process. Every instance involves actors that are the users interacting with the various tasks performed during the instance life cycle.

\section{The taxonomy of change operations}

In the development and the deployment of BPMoriented applications, the change seems inevitable. It enrolls in fact, in the usual life cycle of this kind of applications. We define it as a taxonomy of change operations that may affect one of the important components of these applications to know the BPM. We con-

\footnotetext{
5 http://user.cs.tu-berlin.de/ gragra/agg/
}

sider two kind of changes : the atomic change operations and composite or complex change operations.

\subsection{The atomic change operations}

The formalization of a BPM as a typed attributed graph allows us to compile a list of atomic change operations. It is important to notify that, "each change operation corresponds to an insertion, deletion or modification of a node or an edge of this graph". We thus obtain the following change operations :

- Insert a process

- Delete a process

- Modify a process (name, description, etc.)

- Insert a new task

- Delete a task

- Modify a task

- Modify an attribute of the task (name, description, etc.)

- Modify the type of a task (automatic, manual, etc.)

- Insert an event

- Delete an event

- Modify an event (start event to intermediate event, etc.)

- Insert a gateway

- Delete a gateway

- Modify a gateway (condition of application: OR to XOR, etc.)

- Insert an edge or link (between two flow objects)

- Delete an edge between two flow objects

- Et cetera.

Each defined atomic change operation is then formalized by graph rewriting rules. A graph rewriting rule is in fact a production rule where the left and right sides of the rule are graphs. In other words, a production rule that transform a part of the graph which match or corresponds to the left hand side (LHS) of the production by another subgraph represented by the right hand side(RHS) of the production. There are also preconditions called negative or NAC, which specify the need for non existence of certain subgraph for the rule to run.

Visually such a rule can be outlined as in the Fig. 5. This figure depicts the partial creation or insertion of a new task. It shows the three components of a rule called InsertTask which represents the insertion of a task in a process. The LHS of the rule states 


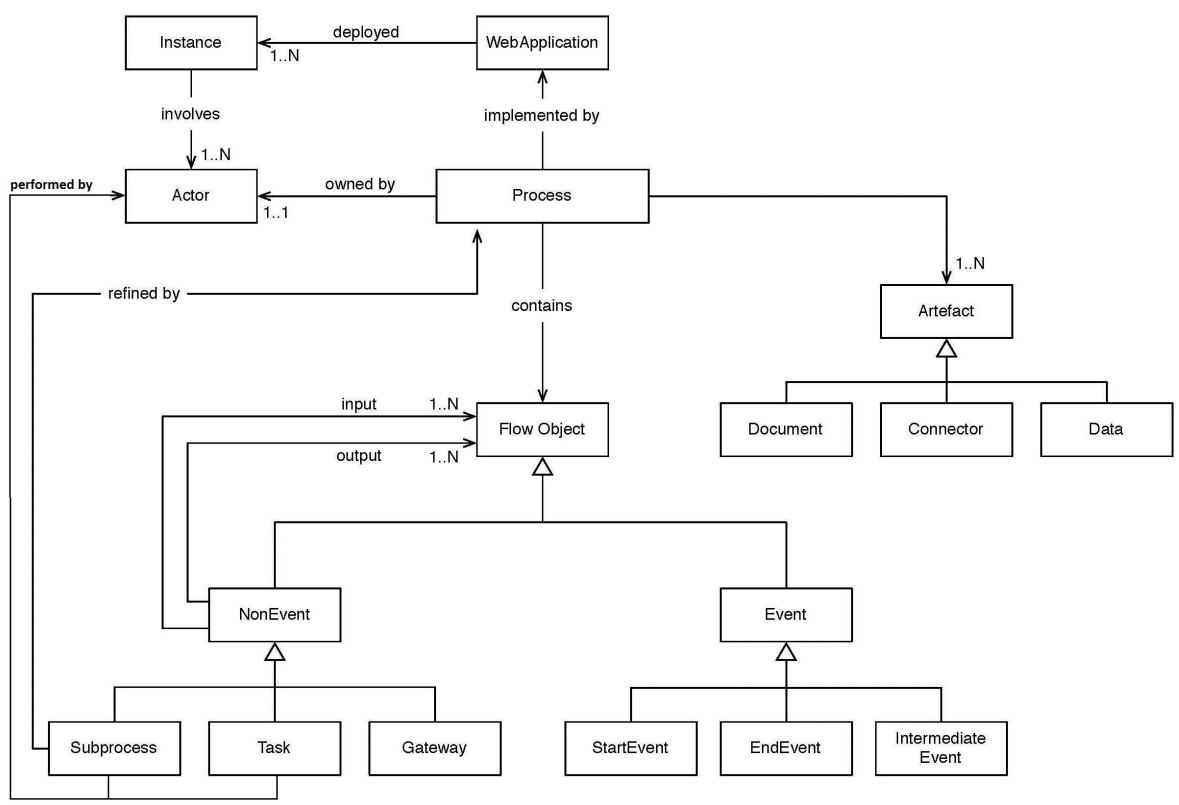

Figure 4: A UML based representation of BPM meta-model

that there must be a node in the graph of process type. It would then match a process node of the graph with that of the rule. This matching can be done manually by the user or automatically by the graph rewriting system following many methods that are out of the scope of this paper. The RHS of the rule shows the creation of a task called $x$ connected to the process by the relationship "contains". The NAC of the rule prohibits the creation of a task named $x$ if there is already a task in the process with the same name.

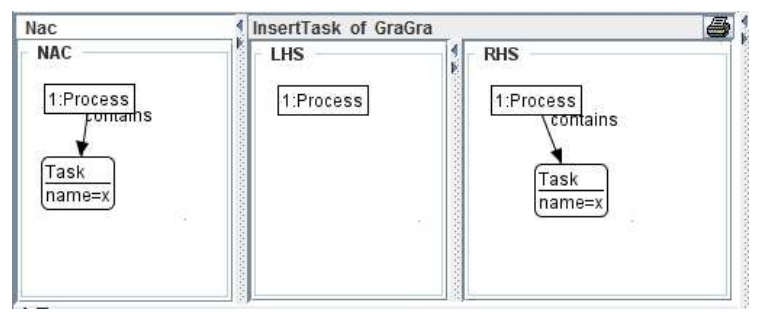

Figure 5: A rule to insert a task

\subsection{The composite change operations}

The composite change operations can be expressed in the form of compositions of atomic operations. We did not set a precise or exhaustive taxonomy of these operations but we consider the most significant and frequent ones. It is also possible to define new composite change operations.

The example of composite change operations :

- The merger of two tasks
- The decomposition of a task into two

- The merger of two processes

- The breaking down of a task into subprocess.

\section{The change impact analysis}

This section deals with the BPM change impact management. A simulation of the change impact generation is presented formerly, using graph rewriting rules and then in later part impact propagation is elaborated to the various artifacts through the different type of relationships.

\subsection{The change impact generation}

All change operations are defined by the preconditions, which in the case of a graph rewriting rules consist of the LHS (positive preconditions) and the NAC (negative preconditions) and the postconditions symbolized by the RHS. We therefore consider the impact of a change $M$ given the result of its execution (symbolized as the RHS), in the case of a violation of the precondition. In the case of graph rewriting rules, this is translated into the creation of a node of Impact type, connected to the nodes affected by the impact and containing an attribute called explanation which contains a narrative of the impact (see Fig. 6). We simulate the creation of the impact by setting rules without NAC and therefore tolerate the enforcement of the rule with the result, in addition to that provided 
by the original rule, appending a node of impact type linked to the nodes it affects. In Fig. 6, we define a rule to delete a task that provokes the creation of an Impact node affecting the tasks related to the task that has been deleted.

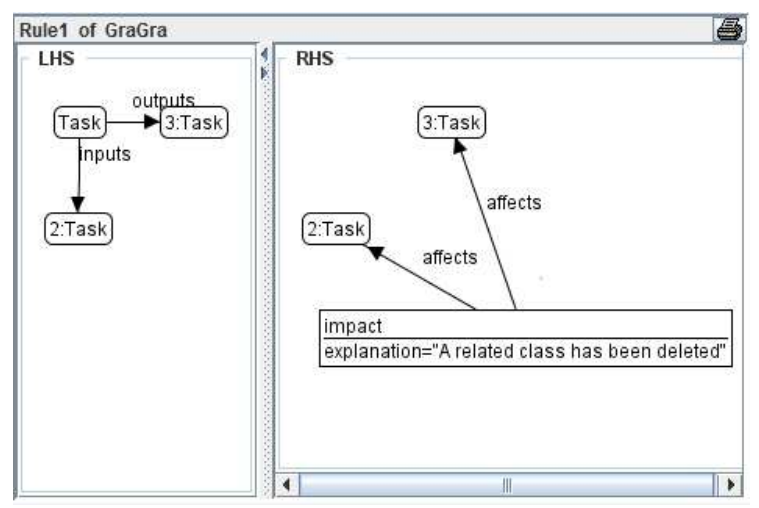

Figure 6: A rule for change impact analysis of a task deletion

\subsection{The change impact propagation}

The change impact propagation is a process of propagating the impact to all nodes indirectly affected by the impact. This propagation is done through a link or relationship between nodes. Thus, some relationships are identified as change impact conductor (Ahmad et al., 2008a) and propagate the impact in one way or another. For example, the Fig. 7 shows the propagation of the impact affecting a task to the author of this task with the associated explanation.

We distinguish here two types of change impact propagations :

- The horizontal change impact propagation is to propagate the change impact between artifacts belonging to the same phase of the development life cycle of an application. This is the case of the rule as shown in Fig. 7. It shows the impact propagation between tasks and actors.

- The vertical change impact propagation corresponds to the change impact flow between artifacts belonging to different phases of the development life cycle of an application. This is the case of the change impact propagation between a task and a web service that implements a part of this task and vice versa.

To show how we deal the vertical change impact propagation, we first define a kind of mapping relationships that are useful for the traceability purpose. These relationships are :

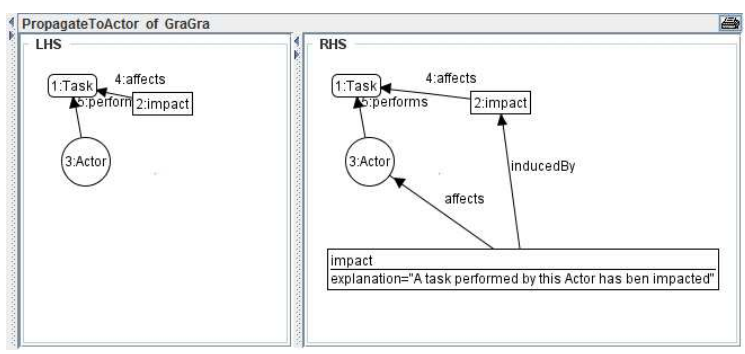

Figure 7: A rule for change impact propagation

- The mapedTo relationship between a BPMN process and a BPEL process. In fact, we defined a meta-model of BPEL processes like we have done with the BPMN but the BPEL process contains objects like web services, etc.

- The ImplementedBy relationship between a task of the BPM and a web service of the BPEL.

We know that this set of mapping relationships is a restrictive one since it is generally possible that a task may be implemented by more than one web service, it may also be implemented by a process. In another hand a BPMN process may be implemented by a set of BPEL, ones. But we just want to demonstrate the feasibility of our approach at this initial stage and then refine it more deeply in the future works.

The mapping relationships are then used by the graph rewriting rules generating or propagating the impact. So, the Fig. 8 shows the impact generated by the composite change consisting of the merging of two tasks. The question here is what to do with web services implementing these tasks?

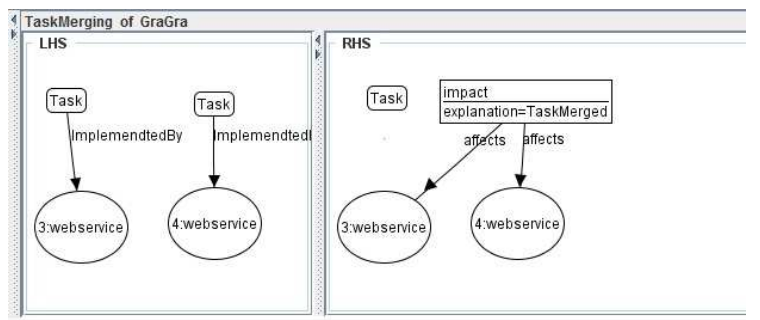

Figure 8: Rule for the impact generated by the composite change

On the other hand, we consider three kinds of change impact propagation processes.

- The total change impact propagation simulates the change operation and then execute all possible rules of its impact propagation.

- The selective change impact propagation only propagates the change impacts induced by a subset of nodes relationships.

- The Propagation of type changes-andfix (Rajlich and Gosavi, 2004; $\quad$ Rajlich, 1997) 
which is to simulate a change, directly addresses the impact of this operation (in terms of direct neighbours). This treatment or correction of the change impact will be a transaction which itself will directly impact the address, and so on.

\section{The prototype of validation}

We use the graph rewriting system as a mean to formally describe the BPM change operations. We also use such systems as an execution engine to evaluate the different strategies we may adopt for the impact management. For an operational validation of the proposed change impact analysis and meta-model, we implement an integrated platform prototype. It allows friendly experimentations of graph structures associated to a software development project. Our platform, named as Architect is a change impact analysis tool for distributed software applications. It is built as a set of Eclipse ${ }^{6}$ IDE plug-ins. An Eclipse project manages a set of resources that can be source code files, libraries, BPEL, and BPMN files etc. Architect analyzes these heterogeneous sources and parses their elements to represent them as a homogenous interactive graph. The Architect Graph extension of eclipse visualizes the elements of the corresponding editor view. This prototype contains a graph editor which provides in a very simple way the nodes and arcs of the structural graph.

We have used the Java Universal Network/Graph $(J U N G)^{7}$ Framework. This is a software library that can be re-used for the modeling, analysis, and the visualization of data as a graph or network. This library allows to define the structure of data Graph and also to use certain graph primitives for the construction of user interfaces associated with the graph manipulation tools. We used it in interaction with the built-in capabilities of Java API, as well as those of other existing third party Java libraries i.e Drools ${ }^{8}$. We have specialized the class Graph available in the JUNG library in a class that we called ArchitectGraph. The large software related information can be stored and manipulated through a semi-automated knowledge-based system. The Drools is a business logic platform which provides an integrated unified platform for the graph rewriting rules, workflow and the event processing. We use it for writing dynamic expert rules as well as general rules of propagation to improve the interactivity of generated graph.

\footnotetext{
6 http://www.eclipse.org/

${ }^{7}$ http: // jung. sourceforge.net/

8 http: //www.jboss.org/drools/
}

By using our platform, one can friendly specify a change affecting a node. Which may be a BPM task, a web service specification, or a Java class, etc. The various rules implementing the change impact propagation may then be fired. As a result the new graph is displayed containing nodes of type "impacted" related to the different impacted artifacts along with the explanation of the propagated impact.

\section{Concluding remarks and future work}

In this paper, we present an approach based on a BPM meta-model intended to serve as a BPM artifacts repository data schema. We also defined the initial BPM change operations taxonomy. It involves the formalization of the change and the analysis of its impact propagation by graph rewriting rules. The graph rewriting rules have been implemented with AGG that as a graph rewriting system. This implementation can be considered as an operational or constructive specification.

We observe that it is possible to define a change impact propagation process concerning both the BPM processes artifacts and their implementations which can be BPEL processes, etc. The main concept of our approach is implemented by a set of tools integrated as ECLIPSE plug-ins. These plugins are parts of a more general integrated framework which is built to deal with the software artifacts change impact propagations.

We are continuing the work by enriching the approach, in a more detailed way, with the different kind of mapping relationships related to the BPM artifacts and their implementation. The main goal is to be able to automatically track the change impact propagation. To achieve this, we must explicitly represent knowledge concerning the semantic of BPM elements and their relationships. We are then using the BPMN ontology (Penicina, 2013) that is expressed using the owl language (Motik et al., 2012). We plan to enrich this ontology by concepts representing the various aspects of change impact and the relationships propagating such impacts. The use of the owl language makes it possible to define, in an explicit way, the relationship hierarchy based on the impact propagation. It is also possible to explicitly define some semantical characteristics of relationships like transitivity, etc. The reasoning capabilities provided by the ontology languages (like owl) may assist the change experts to define change impact rules.

Another goal is to provide some forward and reverse engineering tools in order to implement some 


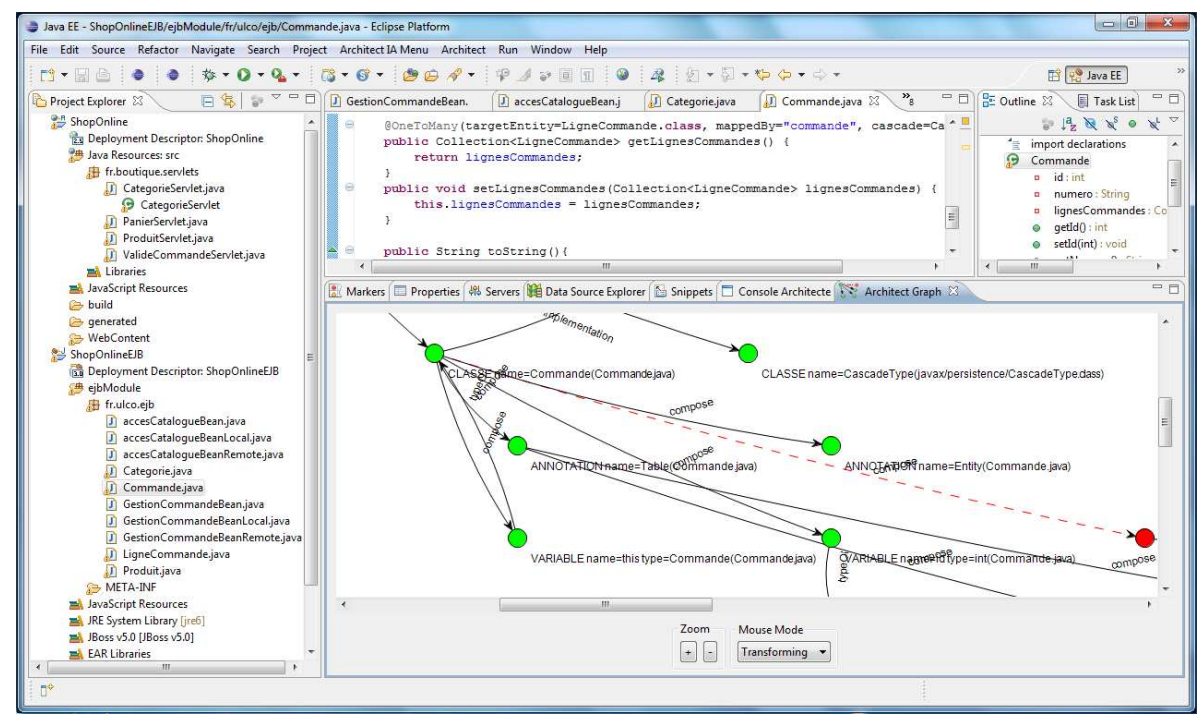

Figure 9: Screen shot of a change impact propagation scenario in Architect

important tasks like defining flexible and adaptable tools for the generation of BPM implementations and some others for the generation of BPMs from the process implementations.

\section{REFERENCES}

Ahmad, A. and Basson, H. (2009). Software evolution modelling: an approach for change impact analysis. In Proceedings of the 7th International Conference on Frontiers of Information Technology, FIT '09, pages $56: 1-56: 4$, New York, NY, USA. ACM.

Ahmad, A., Basson, H., and Bouneffa, M. (2008a). Software evolution control: Towards a better identification of change impact propagation. In ICET'08: Proceedings of the 4th IEEE International Conference on Emerging Technologies, pages 286-291. IEEE Computer Society.

Ahmad, A., Basson, H., and Bouneffa, M. (2009). Rulebased approach for software evolution management. In IEEE APSSC 2009: IEEE Asia-Pacific Services Computing Conference.

Ahmad, A., Basson, H., Deruelle, L., and Bouneffa, M. (2008b). Towards a better control of change impact propagation. In INMIC'08: 12th IEEE International Multitopic Conference, pages 398-404. IEEE Computer Society.

Allweyer, T. (2010). BPMN 2.0 : Introduction to the Standard for Business Process Modeling. Books on Demand, Norderstedt.

Dadam, P., Reichert, M., Rinderle, S., Jurisch, M., Acker, H., Pser, K. G. A., Kreher, U., and Lauer, M. (2007). Adept2 - next generation process management technology. In Proceedings Fourth Heidelberg Innovation Forum, Aachen. D.punkt Verlag.
DeRemer, F. and Kron, H. (1975). Programming-in-the large versus programming-in-the-small. SIGPLAN Not., 10(6):114-121.

Dumas, P., Charbonnel, G., and Calmes, F. (1990). La méthode OSSAD - Pour maîtriser les technologies de l'information - Tome 2: Guide pratique, Les Editions d'Organisation, Paris.

Emig, C., Momm, C., Weisser, J., and Abeck, S. (2005). Programming in the Large based on the Business Process Modeling Notation. In Jahrestagung der Gesellschaft für Informatik (GI), Bonn.

Gottschalk, K., Graham, S., Kreger, H., and Snell, J. (2002). Introduction to web services architecture. IBM Syst. J., 41:170-177.

Haller, A., Gaaloul, W., and Marmolowski, M. (2008). Towards an xpdl compliant process ontology. In SERVICES I, pages 83-86.

Juric, M. B. (2006). Business Process Execution Language for Web Services BPEL and BPELAWS 2nd Edition. Packt Publishing.

Lee, Y.-C., Chu, P.-Y., and Tseng, H.-L. (2011). Corporate performance of ict-enabled business process reengineering. Industrial Management and Data Systems, 111(5).

M.O., H., Deruelle, L., Basson, H., and Ahmad, A. (2010). A change propagation process for distributed software architecture. In ENASE 2010: Proceedings of the 5th International Conference on Evaluation of Novel Approaches to Software Engineering.

Motik, B., Grau, B. C., Horrocks, I., Wu, Z., Fokoue, A., and Lutz, C. (2012). Owl 2 web ontology language: Profiles. w3c recommendation (27 october 2009).

OMG (2011). Business process model and notation (bpmn) version 2.0. OMG Document Number: formal/2011-01-03, Standard document URL: http://www.omg.org/spec/BPMN/2.0 Accessed 2011-03-18. 
Parmenter, D. (2007). Key Performance Indicators (KPI): Developing, Implementing, and Using Winning KPIs. John Wiley \& Sons, Inc., New York, NY, USA.

Penicina, L. (2013). Choosing a bpmn 2.0 compatible upper ontology. In The 5th International Conference on Information, Process, and Knowledge Management, pages 89 - 96, Nice, France. IARIA.

Rajlich, V. (1997). A model for change propagation based on graph rewriting. In Proceedings of the International Conference on Software Maintenance, pages 84-91, Washington, DC, USA. IEEE Computer Society.

Rajlich, V. and Gosavi, P. (2004). Incremental Change in Object-Oriented Programming. IEEE Softw., 21(4):62-69.

Schmidt, D. (2006). Guest editor's introduction: Modeldriven engineering. Computer, 39(2):25 - 31 .

Silver, B. (2009). BPMN Method and Style: A levels-based methodology for BPM process modeling and improvement using BPMN 2.0. Cody-Cassidy Press.

Van der Aalst, W. M. P. (2003). Patterns and XPDL: A critical evaluation of the XML process definition language. Technical Report BPM-03-09, BPMcenter.org.

Weske, M. (2007). Business process management concepts, languages, architectures. Springer, 1 edition.

Weske, M. (2012). Business process management architectures. In Business Process Management, pages 333371. Springer Berlin Heidelberg. 